\title{
Refining the timing of recombination rate plasticity in response to temperature in Drosophila pseudoobscura
}

Authors: Ulku H. Altindag ${ }^{1}$, Chelsea Shoben ${ }^{2}$, and Laurie S Stevison ${ }^{1, *}$

Affiliations: ${ }^{1}$ Department of Biological Sciences, Auburn University, Auburn, AL 36849, USA, ${ }^{2}$ Biology Department, Duke University, Durham, NC, 27005, *Corresponding Author

Data Availability:

Github Repository: https://github.com/StevisonLab/Peak-Plasticity-Project

DOI: $10.5281 /$ zenodo.3948807

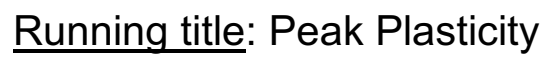

Keywords: meiosis, crossover, heat stress

Corresponding Author:

Laurie Stevison

101 Rouse Life Sciences

Auburn, AL 36849

334-844-1636

Iss0021 at auburn dot edu

\section{Abstract}

Meiotic recombination rates vary in response to environmental factors like temperature. Variation in recombination generates an additional source for genetic variation while errors in this pathway can lead to chromosome nondisjunction. Estimating duration and sensitivity of a meiotic response to environmental perturbation requires an understanding of molecular events and well-designed experimental approaches. An earlier study suggested that the peak (most sensitive) timing of plasticity in Drosophila melanogaster occurred during the pre-meiotic interphase where 
DNA replication takes place in S-phase. Recently, heat stress has been shown to reveal plasticity in recombination rates in D. pseudoobscura. Here, a combination of molecular genotyping and a series of recombination analyses through visible phenotypic markers were used to determine peak plasticity timing in this species. Mutant flies were reared in either control or stress temperatures in a traditional cross design. Using mixed model analysis, the odds of crossover formation was $1.55 \mathrm{X}$ higher during days $7-9(p=0.0017)$ and $1.41 \mathrm{X}$ higher on day $9(p=0.034)$ post-mating in high temperature as compared to control crosses, suggesting this time period as the timing of peak plasticity. Time of peak plasticity at day 9 in $D$. pseudoobscura can be explained by comparison to the model organism D. melanogaster due to similar timing of key meiotic events. This comparative approach will enable future mechanistic work on the duration and the effects of temperature stress on recombination rate.

\section{Introduction}

Meiosis is fundamental for sexually reproducing organisms to generate haploid gametes. This process helps to maintain the correct number of chromosomes in the next generation, critical for gamete viability. Additionally, crossing over during meiosis creates novel genetic variation by recombining parental haplotypes, which can have important consequences for adaptation of species (Charlesworth \& Barton, 1996; Page \& Hawley, 2003).

Early studies in Drosophila have shown that crossover rates vary as a result of various factors including maternal age (see companion paper Pownall et al., 2020), starvation, as well as external humidity and temperature (Plough, 1917, 1921; Bridges, 
1927). Over the last century, other model systems have replicated these results (reviewed in (Parsons, 1988; Agrawal et al., 2005; Stevison et al., 2017)). While perturbations have consequences on events throughout meiosis such as in synaptonemal complex and double strand break (DSB) formation, early meiosis appears to be most sensitive to perturbation (Stevison et al., 2017). Experimental evidence points to temperature sensitive, pre-meiotic interphase as the stage when recombination rate's plasticity is the highest. This coincides with the relationship between meiotic recombination and DNA replication at S-phase (a component of interphase) (Grell, 1973, 1978b).

While there has been a century of work on recombination rate plasticity in $D$. melanogaster, there have been no efforts to document this phenomenon in other Drosophila species. Drosophila is an extremely diverse genus made up of over 2000 species that diverged over 50 million years ago (Hales et al., 2015). Some have argued that Drosophila species can serve as indicators of global climate change (Parsons, 1991). One concern with focusing on D. melanogaster in the study of how environmental stress impacts recombination is that it is a cosmopolitan species, and thus may not have the same environmental sensitivity as other species within the Drosophila species group. Our team has recently worked to expand research on this ubiquitous phenomenon into D. pseudoobscura (Stevison et al., 2017).

While traditionally studied for their inversion polymorphisms, $D$. pseudoobscura is native to western North America and a small region in Bogota, Colombia. It is therefore alpine over parts of its range, which means it has the potential to be more sensitive to environmental changes (Kuntz and Eisen, 2014). Additionally, D. pseudoobscura 
females exhibit synchronization of oogenesis across egg chambers (Donald and Lamy, 1938), which is key to studying the timing of events in meiosis because time is an indicator of progression through oocyte development. More recently, there has been a burst of interest in studying recombination rates in this species (Kulathinal et al., 2008, 2009; McGaugh et al., 2012; Stevison and Noor, 2010; Samuk et al., 2020).

Our lab recently reported a preliminary analysis of recombination rate plasticity due to heat treatment during development in D. pseudoobscura (Stevison et al., 2017). In this study, significant plasticity was found in eight regions across the 2nd chromosome, with 5/8 regions showing higher recombination in the high temperature treatment (see Table S1 in Stevison et al 2017). These results parallel work done in $D$. melanogaster (Grell, 1966, 1973, 1978a). In addition to temperature, the impact of maternal age on recombination rates in D. pseudoobscura was investigated (see companion study Pownall et al., 2020).

Here, this work was continued to establish D. pseudoobscura as a model for studying recombination rate plasticity. Temperature was used as treatment throughout development similar to work of Plough and others (Plough, 1917, 1921; see Figure 2 in Stevison et al., 2017). A series of experiments were conducted with the goal of pinpointing the timing of peak differences in recombination rate between control and temperature stress crosses. First, SNP genotyping markers were initially used to confirm the suitability of using phenotypic mutants to study recombination rate plasticity. Next, five experiments using phenotypic mutants were conducted where the experimental parameters were adjusted with each successive experiment, altering treatment temperature, duration of progeny collection, progeny transfer frequencies, 
and sample sizes (summarized in Table 1; Figure 3). The first two experiments were inconclusive, but the third experiment revealed that the time point of 7-9 days postmating had $5.6 \%$ difference between control and temperature stress crosses. However, it was discovered that the phenotypic mutant thought to be vermillion was in fact associated with the scarlet gene, which is $52 \mathrm{cM}$ from the yellow mutant marker. A fourth experiment similar to the third, but with a new mutant stock, failed to uncover the 7-9 day peak in plasticity. However, the fifth experiment using these new markers and a 24hour transfer frequency revealed day 9 to have $8.3 \%$ difference in recombination rates in the sd-y interval (Figure 4). Therefore, the results of experiments 3 and 5 were most influential in overall conclusions (see Discussion), and the others served to guide changes in experimental design. Combining strategies used in earlier studies, the work presented here will be essential to future mechanistic work to understand the mechanism of recombination rate plasticity and enable it to be studied in more depth in D. pseudoobscura.

\section{Materials and Methods}

\section{Stocks}

Recombination analysis using mutant markers were conducted using two Xlinked recessive mutant $D$. pseudoobscura stocks. First, a double mutant stock was produced by crossing two lines obtained from the U.C. San Diego stock center (which has relocated to Cornell University): vermilion ( $v$; 1-88.3) (stock 14011-0121.06, Dpselv[1]) and yellow (y; 1-74.5) (stock 14044-0121.09, Dpsely[1]). Analysis has shown that the vermillion stock is most likely scarlet (see Results). Mutations of the vermilion 
(and scarlet) gene induce a bright red-eye phenotype, and mutations within the yellow gene induce a yellow-hued body and wings. Second, a quadruple mutant stock (courtesy of Nitin Phadnis) was used that had four mutations - yellow ( $y$; 1-74.5), cut (ct; 1-22.5), scalloped (sd; 1-43.0), and sepia (se; 1-156.5) (Phadnis, 2011). Mutations of the cut and scalloped genes induce changes to the wing phenotype, whereas mutations in the sepia gene result in brown eyes. Genetic locations of all mutant markers are shown in Figure 1B. The ct marker produced inconsistent results likely due to a variation in penetrance of the cut mutation (Dworkin et al., 2009). Therefore, the ct$s d$ region was excluded from the remainder of the analysis.

Three wildtype $D$. pseudoobscura stocks were also used for genetic crosses. First, MV2-25 was used in crosses to the double mutant stock, which are both in an Arrowhead 3rd chromosome background. Second, AFC-57 (see (Ritz et al., 2017)) was used for indel genotyping. Finally, to match the 3rd chromosome inversion arrangement of the multiple marker line, a second stock bearing the arrangement called "Treeline" was obtained from the National Drosophila species Stock Center at Cornell University (stock 14011-0121.265, Dpselwild-type "TL", SCI_12.2).

\section{Husbandry \& Cross Design}

All stocks were maintained at $21^{\circ} \mathrm{C}$ with a 12 hour light-dark cycle in an incubator. Flies were reared on standard cornmeal-sugar-yeast-agar media in polypropylene enclosures.

For indel genotyping, all crosses were performed at $20^{\circ} \mathrm{C}$ in glass vials containing $6 \mathrm{~mL}$ of corn syrup food. Virgin female $y v$ flies (5-7 days old) were crossed with male AFC-57. Virgin $\mathrm{F}_{1}$ females (5-7 days old) were collected and crossed with $y v$ 
male flies. Resulting backcross progeny were phenotyped. Cross design for the SNP genotyping markers was described elsewhere (Stevison et al., 2017).

For crosses to measure recombination rate, double and quadruple mutant stock virgins were collected and aged 7 days to full sexual maturity. These flies were crossed to wild-type, age-matched, Treeline arrangement males in control conditions to produce heterozygous $F_{1}$ progeny (Table 1 ). After crosses, vials were assigned to identical incubators with a 12 hour light-dark cycle with the temperature varying according to Table 1 (see below). Virgin heterozygous $F_{1}$ females were aged to 7 days and backcrossed to wild-type males from $21^{\circ} \mathrm{C}$ following single male, single female matings. However, for Experiment 3, the backcross was done to the mutant stock (see below). Crossing schemes for recombination analysis are diagrammed in Figure 2 with details on each experimental design outlined in Table 1 and below. Before backcrosses, wildtype males were individually isolated 24 hours prior to crosses to avoid crowdinginduced courtship inhibition (Noor, 1997). After 24 hours, the wild-type males were discarded to prevent additional stress from male harassment (Priest, 2007). The female continued to be transferred to a fresh food vial according to the transfer frequency of each experiment (Table 1).

Additionally, the vials where virgins were held prior to genetic crosses were kept for 14 days to ensure there were no larvae. If larvae were found, the cross was discarded. To increase sample sizes, multiple backcrosses were conducted from each replicate $F_{1}$ female. 


\section{Experimental Design}

For each successive experiment, transfer frequency and duration were varied to pinpoint the timing of peak differences in recombination rate due to temperature (see Table 1). A series of five experiments were conducted using double (1-3) and quadruple (4-5) mutant stocks. First, in Experiment 1, the experimental design was varied from the SNP genotyping experiment (as described in Stevison et al., 2017) by extending the collections from 10 days to 15 days and second, by conducting 24 hour transfers.

Temperature was also increased from $23^{\circ} \mathrm{C}$ to $24.5^{\circ} \mathrm{C}$ to maximize differences between the treatment and control (Table 1), careful to avoid going beyond upper bounds of developmental temperatures reported in the literature of $25^{\circ} \mathrm{C}$ (Kuntz and Eisen, 2014). In Experiment 2, based on the differences at the later time points of days 10-12 and 1315 in the first experiment (see results), the time frame was extended even further from 15 to 20 days, but considerably widened the transfer frequency from 24 hours transfers to 120 hour transfers (Figure 3B). The control temperature was shifted from $18^{\circ} \mathrm{C}$ to the more standard rearing environment of $20^{\circ} \mathrm{C}$ (Smith, 1958). To maintain a $5^{\circ} \mathrm{C}$ difference between treatment and control, the temperature treatment was also shifted slightly to $25^{\circ} \mathrm{C}$ (Table 1). Because the first two experiments failed to yield significant differences (see results), the cross design for the third experiment was altered to maximize sample size. Specifically, backcrosses were conducted to the X-linked recessive mutant stock rather than the wildtype stock as in Experiments 1-2, allowing for the inclusion of female progeny in recombination calculations. Additionally, transfers were selected based on the aggregation of Experiment 1 data to hone in on the earlier time points with 48 hour 
transfers for the first 6 days and 72 hour transfers for the remaining 9 days, for 15 days total.

Using the quadruple mutant stocks, the design of Experiment 4 closely matched Experiment 3 to replicate the findings of the successful experiment (see results). The transfer frequencies were modified to 72 hours for simplicity. Additionally, because there was no effect of temperature on fecundity in experiments at $25^{\circ} \mathrm{C}$, the temperature treatment was increased to $26^{\circ} \mathrm{C}$ to increase the temperature stress. In Experiment 5, the 7-9 day time period was honed in with 24 hour transfers. However, to maximize the sample sizes in the later time points, both the number of replicates and crosses were increased. Additionally, the vials where females were held for the first five days were discarded to keep the total sample size manageable.

\section{$\underline{\text { Recombination Analysis Using Phenotypic Markers }}$}

Resulted progeny were screened for recombination rates differences between treatment and control conditions (Table 1). Except for Experiment 3, only male progeny were scored and if any female progeny were found to be mutant, the entire vial was discarded and the data removed. Recombination analysis using mutant markers recorded each of the mutant traits independently in a single-blind manner. For Experiments 4-5, mutant scoring was delayed at least 5 days for the sepia eye color to become more pronounced. Phenotyping ended 2 weeks after eclosion started to prevent the next generation from being included in the data. Every effort was made to score all progeny. Data were entered in triplicate and compared until $100 \%$ concordant. 


\section{Molecular Genotyping}

As part of a preliminary characterization of plasticity in $D$. pseudoobscura, Sequenom SNP genotyping markers were designed to genotype crosses between FS14 and FS16 wildtype flies (methods described previously, see Stevison et al. 2017). These results capture chr2. In addition, for this study, seven additional SNP markers were designed on the left arm of the $\mathrm{X}$ chromosome $(\mathrm{chr} \mathrm{XL})$ to span the region containing the mutant markers yellow and vermillion (Figure 1A). One marker was excluded based on screens of $F_{1}$ progeny, where the marker was completely homozygous, leaving six total. Together, the five intervals span $5 \mathrm{Mb}$ of the $\mathrm{XL}$ and are located on scaffold chrXL_group1e of the $D$. pseudoobscura reference genome.

Additionally, molecular genotyping was used to confirm association between phenotypic mutants and their respective genes for the yellow and vermillion genes. For this analysis, two indel markers were designed based on the D. pseudoobscura assembly v3.1, each within $25 \mathrm{~kb}$ from the vermilion and yellow genes. Markers selected resulted in differing PCR product length between the mutant stocks and the wild type AFC-57 stock (Table S1). DNA was isolated (Gloor and Engels, 1992) from a minimum of 88 flies for each parent stock and backcross progeny for PCR amplification. Length differences for markers were assayed via acrylamide gel. To confirm linkage between the vermilion and yellow genes and the red eye and yellow body phenotypes, backcross progeny of known phenotype were genotyped for the vermilion-linked and yellow-linked indel markers. 


\section{Statistical Analysis}

Statistical analysis was performed using R v4.0.1 (R Core Team, 2020).

Recombination rate frequencies were calculated for the chromosomal interval between each phenotypic marker (Figure 1A). Recombination frequencies (RF) correlating mapping distance between linked alleles were calculated by dividing the number of recombinant flies for regions $y-v, s d-y$, or $y$-se to the total number of progeny.

GImer function was used to generate a fitted generalized linear mixed-effects model (GLMM) per interval with replicate vial IDs as random effects and all other parameters as fixed effects. For each interval within each experiment, a logistic regression analysis with a mixed model was conducted in $\mathrm{R}$ (Supplementary Tables 14). The basic model equation was:

$$
\text { Equation 1: } \quad \mathrm{R}=\mathrm{V}+\mathrm{D}+\mathrm{T}+\mathrm{D}^{*} \mathrm{~T}
$$

' $R$ ' indicates the binary response variable of whether an individual offspring was recombinant or not based on the pair of mutant phenotypes over the screened region. 'V' indicates the replicate vial ID and corresponds to $F_{1}$ crosses. Progeny from backcrosses of $F_{1}$ female siblings were summed per replicate cross per day and any replicate with fewer than 10 progeny were removed to avoid stochasticity in recombination rate estimates. ' $D$ ' indicates days post-mating of the $F_{1}$ female. Finally, ' $T$ ' indicates the temperature in which the $F_{1}$ female was reared. For the most part, interaction terms were not significant, so individual odds ratios were extracted for each time point (Figure 3). For logistic regression, exponentiating the coefficients of GLMM generates the odds of crossover formation between experimental and control conditions. 
Additionally, fecundity was tracked to measure the impact of stress due to temperature treatment was calculated by dividing the number of backcross progeny to the number of $F_{1}$ mothers. Linear regression analysis was conducted following a similar basic model equation:

Equation 2: $\quad F=V+D+T+D^{*} T$

Here, all variables are the same as Eq. 1, except the response variable, 'F', in this model is the total number of progeny, fecundity, for each time point. For each replicate cross, fecundity was summed over all crosses and divided by the number of crosses per replicate to get an average number of progeny per time point for each replicate.

The results of both models are summarized in Table 1 and Supplementary Tables 1, 2, and 5. Additionally, a post hoc Ismeans contrast was conducted to compute the significance of treatment versus control for each time point in each experiment (see Supplementary Tables 3, 4, and 6).

\section{Data Availability}

Data files and scripts to complete the analysis are available on github at https://github.com/StevisonLab/Peak-Plasticity-Project. A snapshot of the code at the time of publication is available at DOI: $10.5281 /$ zenodo.3948807. Also, a walk through tutorial for the analysis of data in Experiment 3 in $\mathrm{R}$ markdown has been included. Sanger sequence data for $179 \mathrm{bp}$ of the scarlet gene, capturing the $2 \mathrm{bp}$ deletion, in the bright red eyed flies (originally ordered vermilion stock) is available on NCBI (GenBank accession number MT438819). 


\section{Results}

\section{SNP genotyping markers confirm recombination plasticity of region spanning phenotypic markers}

In an earlier molecular analysis, results were described for markers on the 2 nd chromosome (Stevison et al., 2017). That analysis also included six X-chromosome SNP genotyping markers in the region spanning the genes yellow and vermillion (Figure 1A). In analyzing crossover data for intervals 1-3 (shown in black in Figure $3 A$ ), the results show that control crosses had a $12.2 \%$ recombination rate, similar to the documented recombination fraction of 14.6 (Anderson, 1993). The high temperature crosses had a $16 \%$ recombination rate across the same three intervals, which was significantly higher than the control $(p=0.019)$. Across the five intervals, a significant difference due to temperature was observed for interval 4 (i4 in Figure 3A), between markers $m_{4}$ and $m_{5}$, and a significant interaction term was observed for intervals 2 and 3 (Supplementary Table 1). Additionally, a post hoc means contrast between treatment and control revealed a significant difference in recombination frequency $(R F)$ in intervals 3 and 4 (Supplementary Table 3). Specifically, interval 3 differed on days 5-6, and interval 4 differed on days 3-4. Both intervals 3-4 had a significant peak difference on days 9-10 (Figure 3A). These results confirmed the suitability of this region to use phenotypic mutants to refine the timing of recombination rate plasticity in response to temperature stress. 
Initial recombination analysis using double mutant stock narrows plasticity to $7-9$ days

A series of three experiments were conducted to pinpoint the timing of differences in recombination rate by crossing a double mutant stock (yellow and "vermillion") to the wildtype genome line MV2-25 (Figure 2A). These experiments varied in transfer frequency and duration of progeny collection (Table 1). It was later discovered that the red eye mutant was associated with the scarlet gene rather than the vermillion gene, which explained differences in expected recombination frequencies in these experiments (see below).

In Experiment 1, several time points had a low sample size with too few replicates and missing data for either treatment or control. Therefore, time points were aggregated for analysis (Figure 3B). Still, recombination differences when aggregating all time points $\left(\mathrm{RF}_{18}=44.3 \% ; \mathrm{RF}_{24.5}=46.7 \%\right)$ were not statistically different. Similarly, Experiment 2 did not show any statistically significant differences in RF in any individual time points (Figure $3 \mathrm{C}$ ), or when aggregated $\left(\mathrm{RF}_{20}=44.7 \% ; \mathrm{RF}_{25}=47.8 \%\right)$. Additionally, due to the experiments having only a single mating event, the progeny sample sizes for days 16-20 in Experiment 2 were too small for further consideration.

Experiment 3 was $5 x$ and $8 x$ larger than Experiments 1 and 2, respectively $(\mathrm{N}=9,693)$, due to switching the cross design (Figure $2 \mathrm{~A}$, red asterisk). This resulted in a significant treatment effect in the statistical model (see Eq. 1; $p=0.011$, Table 1;

Supplementary Table 2). Additionally, in a post hoc means contrast between treatment and control, days 7-9 had statistically significant higher odds of observing a crossover at high temperature $\left(p=0.0017 ; \mathrm{N}_{20}=466 ; \mathrm{N}_{25}=407\right)$ with $\mathrm{RF}_{25}=55.4 \%$ and $\mathrm{RF}_{20}=45.7 \%$ 
(Figure 3D; Supplementary Table 4). Additionally, days 13-15 were significantly different, but with a large standard error (see Supplementary Table 4) and small sample size with fewer replicates $\left(\mathrm{N}_{20}=387 ; \mathrm{N}_{25}=99\right)$. Thus, moving forward, the collection period was reduced to 12 days and focused on days 7-9.

Rearing temperature did not significantly explain differences in fecundity (Eq. 2) across Experiments 1-3 (Table 1; Supplementary Table 5). However, Experiment 1 and 3 showed a decrease in the mean fecundity between temperatures of $17 \%$ and $19 \%$ respectively (Figure S1).

\section{Molecular genotyping reveals vermillion mutant is actually scarlet}

In the experiments described above, the recombination fraction calculated between the red eye and yellow body phenotypes were $46 \%$, much higher than the published $14.6 \%$, indicating that one of the mutants was not in the expected gene region. For the indel marker near the yellow gene, results indicated an association between genotype and phenotype (recombination fraction 0/88). However, upon genotyping an indel marker near the vermillion gene, it was discovered that the bright red eye phenotype did not associate with the indel marker among backcross progeny (recombination fraction 39/85).

Next, a mutation in scarlet (st), another X-linked marker in D. pseudoobscura, which also causes a red-eye phenotype was investigated. Genotyping confirmed that the bright red eye phenotype was $100 \%$ associated with an indel within $21.9 \mathrm{~kb}$ of the (st) gene (recombination fraction 0/471). Sanger sequence analysis revealed a $2 \mathrm{bp}$ deletion within the scarlet gene of the bright red eyed flies of this strain, suggesting a frame-shift mutation within the scarlet gene is a likely cause for this phenotype. 
Although there is not a consistent map location for scarlet in the literature in $D$.

pseudoobscura (Beers, 1937; Mampell, 1943), it is consistently 30cM away from sepia

(Figure 1B). This places it roughly 52cM away from yellow, consistent with the observed recombination rates in Experiments 1-3.

Recombination analysis using multiple mutant stock pinpoint 9 day peak in plasticity

Once the double mutant stock was confirmed to contain markers that were $>50 \mathrm{cM}$ apart, a quadruple mutant stock was obtained to continue recombination analysis (Figure 2B). Two additional experiments were conducted to refine the timing of recombination rate plasticity. Results for the $s d-y$ region $(32.1 \%)$ closely matched the expected rate $(32.5 \%)$. Similar to $y$-st, the $y$-se region had a large recombination rate $(46.0 \%)$ consistent with the genetic map distance $(82 \mathrm{cM})$, since markers over $50 \mathrm{cM}$ apart have a $50 \%$ recombination frequency. However, the results for the $c t-s d$ region (6\%) were substantially lower than expected $(20.5 \%)$, thus the $c t$-sd region was excluded from further analysis (see Methods).

For Experiments 4-5, the treatment did not significantly explain recombination rate in either interval for the overall model (Eq. 1; Table 1; Supplementary Table 2). A post hoc means contrast between treatment and control for Experiment 4 revealed that the highest odds ratios were observed on days 4-6 for the sd-y region (1.24) and on days $10-12$ for the $y$-se region (1.107, Figure $3 \mathrm{E})$, though none of these results were significant (Supplementary Table 4). In Experiment 5, the highest odds ratio was observed on day $9(1.41)$ in the $s d-y$ region (Figure 3F, $p=0.034, N_{21}=630$ and 
$\mathrm{N}_{26}=296$ ). Similarly, the median RF across replicates for this time point was $32.1 \%$ at $21^{\circ} \mathrm{C}$ and $40.4 \%$ at $26^{\circ} \mathrm{C}$ (Figure 4). While the results for this time point were not significant for the $y$-se interval, there was a $2.58 \%$ difference in RF, indicating a similar effect on this time point. When the data for both intervals are combined, there was a $10.97 \%$ difference in total recombination on day 9 .

In Experiments 4-5, temperature had a significant effect on fecundity (Table 1, Supplementary Figure 1; Supplementary Table 5), with a decrease in the treatment group indicating the stress response from the higher temperature of $26^{\circ} \mathrm{C}$. For Experiment 4 , there was a $51 \%$ decrease in mean fecundity due to temperature $(p<0.0001$, see Table 1; Supplementary Table 6). In Experiment 5, average fecundity from days $6-10$ in the control and experimental conditions were 20.9 and 15.0, respectively $(p<0.019)$.

\section{Recombination rates for days 7-9 is reproducible between experiments}

Due to the variation in experimental parameters across experiments, a direct comparison is not possible between all experiments. However, the same time points from the same regions can be compared for a subset of experiments. Specifically, the 79 day time point can be compared between Experiments 1 and $3(y-s t)$, and Experiments 4 and $5(s d-y ; y-s e)$. The raw crossover count data from days 7-9 were aggregated from the 24 hour transfers in Experiments 1 and 5 into the same postmating timeline of 72 hours in Experiments 3 and 4, respectively, for comparison. Odds ratios and standard errors were calculated and compared between individual experiment pairs (Figure 5). For Experiments 4 and 5, the results are highly similar with overlapping confidence intervals for both marker intervals. However, the temperatures 
for Experiments 1 and 3 were different, likely explaining the non-overlapping confidence intervals.

\section{Discussion}

Both Experiments 1 and 2 showed a higher RF in the temperature treatment than the control (Figure 3B-C; n.s.), with Experiment 2 having a slightly larger effect size, likely due to the adjustment to temperature. Although there was not enough power to say whether these were significant differences, likely due to low sample size (Table 1), they were useful in guiding the design of the Experiment 3. Results in Experiment 3 narrowed the timing of recombination rate plasticity in response to heat stress to 7-9 day post-mating (Figure 3D). This result is consistent with the SNP genotyping results, where the 9-10 day time point was significant for 2/5 intervals (Figure 3A). The double mutant stock was then found to have markers that were $52 \mathrm{cM}$ apart rather than the expected 14.6cM distance. Therefore, a quadruple mutant stock was used to further narrow the timing of the peak difference in recombination rate due to temperature as day 9 post mating in Experiment 5 (Figure 3F). On day 9, recombination rate in the $s d-y$ interval was $8.3 \%$ higher in flies reared at $26^{\circ} \mathrm{C}$ as compared to flies reared at $21^{\circ} \mathrm{C}$ (Figure 4), and $10.97 \%$ higher in the total sd-y-se region. Interestingly, declined fecundity due to heat stress was only significantly different in Experiments 4-5 (Figure S1) where the temperature stress was at $26^{\circ} \mathrm{C}$ (maximum of the species range, (Kuntz and Eisen, 2014). Specifically, a significant correlation between fecundity and recombination rate was found among the replicates for day 9 in Experiment 5 (Figure 
$\mathrm{S} 2 \mathrm{~B})$ in control $\left(\mathrm{p}_{\mathrm{Exp} 5}=0.007\right)$. This was not the case for days 7-9 in Experiment 3 (Figure S2A).

Finally, data from experiments using 24-hour transfers were binned to match the day range for data from experiments with $>24$ hour transfers. Comparison between these data confirmed reproducibility. Results between Experiments 4 and 5 were highly concordant and within the $95 \% \mathrm{Cl}$ for both intervals sd-y and $y$-se (Figure S3). However, for the interval $y$-st between Experiments 1 and 3, they were not overlapping, likely due to differences in temperatures between those experiments.

Peak plasticity at day nine points to events in early meiosis

The physiological processes influencing recombination rate need to be further explored. Although there has been a lot of work done in $D$. melanogaster, there are other Drosophila species that may be more sensitive to environmental perturbations for studying this important phenomenon. For example, cactophilic (Markow, 2019) and mushroom feeding (Scott Chialvo et al., 2019) Drosophila represent recent adaptive radiations with growing potential for ecological genomics. Additionally, the montium species group has recently become genome-enabled and is well suited for testing various evolutionary hypotheses (Bronski et al., 2020).

Here, we have examined plasticity in the alpine species, D. pseudoobscura. Exchange between homologous chromosomes starts in the early pre-meiotic stages and are not yielded as crossing over until metaphase I in all eukaryotes (Page and Hawley, 2003). In D. melanogaster, development from oogenesis to egg maturation takes 10 days. Oocyte selection and development during oogenesis occurs in stages 1- 
14 in the last 79 hours (Koch and King, 1966). Although, D. pseudoobscura oogenesis remains understudied, Drosophila species respond to temperature in a distinct manner. Moreover, the decrease in developmental time due to increased temperature is muted in alpine species such as D. pseudoobscura (Kuntz and Eisen, 2014). However, in Experiment 4, a 3-4 day shorter development time was seen in the high temperature treatment as compared to the control. Still, a major benefit of this system is the synchronicity of oogenesis among females that seems to alter with maternal age and indirectly affect fecundity (see Introduction). In D. pseudoobscura, the number of eggs laid indicates a periodicity compared to $D$. melanogaster that continuously lays their eggs in the 12 hours day/night cycles. In D.pseudoobscura, eggs ripen as batches, with the immature eggs divided into groups of differing stages of development, ready to be deposited in large amounts at a time (Donald and Lamy, 1938).

Moreover, crossover formation varies in the same way as different phenotypes are influenced by physiological factors. Developmental events such as invagination of the cephalic fold in stage 8 and retracting of the germband in stage 13 of embryogenesis happen faster in high temperature in $D$. pseudoobscura. The gene recombination defect (rec) was identified by Grell in temperature sensitive mutants of $D$. melanogaster, and is involved in generating meiotic crossovers and DNA double strand break (DSB) formation (Grell, 1984). Later studies in D. pseudoobscura examined the protein encoded by the rec gene, MCM8, which has been shown to be evolutionarily conserved (Grell, 1978b). MCM8 contributes to the stability of DNA strands during DSB and synaptonemal complex formation, and is transcribed early in developmental stages (Hunter, 2015). In Drosophila, these events take place concurrently and affect 
regulation of crossovers (Carpenter, 1975). The protein complexes common in these processes show a temporal pattern that can be tracked by developmental stages.

Grell's work in D. melanogaster showed that all three processes were present in 16-cell cyst in the adult flies by 6 days, pinpointing the peak plasticity at the same time. Grell's studies followed 6 hour transfers to identify the peak timing of recombination due to temperature stress in D. melanogaster, to occur at six days following perturbation (Grell, 1973). While the experimental design in this study is quite different from Grell's work, it is worth noting that in $D$. pseudoobscura, late replication domains indicated with markers of repressive histone marks and SUUR protein are present in the early stages of oogenesis, indicating the pre-meiotic S-phase occurs after day 8 post-mating coinciding with the observed peak in recombination rate plasticity in this study (Grell, 1973; Higgins et al., 2012; Andreyenkova et al., 2013).

\section{Spatial variation in plasticity differs from previous work}

Another interesting finding was that regions closer to the centromere appear to have a peak recombination difference due to temperature later than regions further away (Figure 1A). This finding contrasts previous experiments in $D$. melanogaster, which also showed regions across the chromosome are asynchronous in their timing of peak plasticity, but that regions near the centromere are affected earlier (Grell, 1973). Specifically, the SNP genotyping had a peak odds ratio of 4.99 on days 5-6 for the region between $m_{3}$ and $m_{4}$ (black in Figure $3 A$ ). Whereas, the region between $m_{4}$ and $\mathrm{m}_{5}$ (orange in Figure $3 \mathrm{~A}$ ) had a peak odds ratio of 3.42 on days $3-4$. This finding suggests the timing of plasticity varies spatially along the genome as well as over time, not unlike the findings of Grell (Grell, 1973). It is also worth noting that the magnitude of 
the difference due to temperature was higher for the SNP genotyping experiment than the experiments using phenotypic mutants (Figure 3). It is possible that the larger genetic distance between mutant markers averages out extremes in recombination rate plasticity, leading to a muted overall effect size. A more comprehensive genome-wide study at a fine-scale would be useful to understand both the variation in the magnitude and the spatial variation of this phenomenon.

\section{Acknowledgements}

This work was supported by research start-up funds to LSS from the Department of Biological Sciences at Auburn University. UHA was supported by NSF-DEB EAGER No. 1939090 to LSS. We thank members of the Noor Lab for generating the double mutant $y v$ stock used for Experiments 1-3. We thank Mohamed Noor for guidance and feedback on this work. We thank the Graze lab for help with fly husbandry for Experiments 1-3, which were conducted in the fly lab of Rita Graze of Auburn University. We thank the Phadnis Lab for sharing the quadruple mutant stock used for Experiments 4-5. We thank Todd Steury for consultation on the statistical analysis. We thank members of the Stevison Lab for extensive help with recording mutant marker phenotypes, with particular thanks to Natalia Rivera-Rincon, Keeley Pownall, Neeve Curley, Kyle Meding, Hannah Taylor, Anna Tourne, Adam King, Kaitlyn Walter, and several other undergraduate researchers over a three year period.

\section{Conflict of Interest}

Authors state that there is no conflict of interest. 


\section{References}

Agrawal AF, Hadany L, Otto SP (2005). The Evolution of Plastic Recombination.

Genetics 171: 803-812.

Anderson W (1993). Linkage map of Drosophila pseudoobscura. In: O’Brien SJ (ed)

Genetic Maps: Locus Maps of Complex Genomes, Cold Spring Harbor

Laboratory Press: Cold Spring Harbor, N.Y, pp 3252-3253.

Andreyenkova NG, Kolesnikova TD, Makunin IV, Pokholkova GV, Boldyreva LV,

Zykova TY, et al. (2013). Late Replication Domains Are Evolutionary Conserved

in the Drosophila Genome. PLOS ONE 8: e83319.

Beers CV (1937). Linkage Groups in Drosophila pseudoobscura, Race B. Genetics 22: $577-586$.

Bomblies K, Higgins JD, Yant L (2015). Meiosis evolves: adaptation to external and internal environments. New Phytol 208: 306-323.

Bridges CB (1927). THE RELATION OF THE AGE OF THE FEMALE TO CROSSING OVER IN THE THIRD CHROMOSOME OF DROSOPHILA MELANOGASTER. J Gen Physiol 8: 689-700.

Bronski MJ, Martinez CC, Weld HA, Eisen MB (2020). Whole Genome Sequences of 23 Species from the Drosophila montium Species Group (Diptera: Drosophilidae): A Resource for Testing Evolutionary Hypotheses. G3 Genes Genomes Genet.

Carpenter ATC (1975). Electron microscopy of meiosis in Drosophila melanogaster females: I. Structure, arrangement, and temporal change of the synaptonemal complex in wild-type. Chromosoma 51: 157-182.

Charlesworth B, Barton NH (1996). Recombination load associated with selection for increased recombination. Genet Res 67: 27-41. 
Donald HP, Lamy R (1938). VI.—Ovarian Rhythm in Drosophila. Proc R Soc Edinb 57:

78-96.

Gloor GB, Engels WR (1992). From: Single-Fly DNA Preps for PCR. Drosoph Inf Serv

71: $148-149$.

Grell RF (1966). The Meiotic Origin of Temperature-Induced Crossovers in

DROSOPHILA MELANOGASTER Females. Genetics 54: 411-421.

Grell RF (1973). RECOMBINATION AND DNA REPLICATION I N THE DROSOPHILA

MELANOGASTER OOCYTE. : 22.

Grell RF (1978a). High frequency recombination in centromeric and histone regions of

Drosophila genomes. Nature 272: 78-80.

Grell RF (1978b). Time of recombination in the Drosophila melanogaster oocyte:

evidence from a temperature-sensitive recombination-deficient mutant. Proc Natl

Acad Sci 75: 3351-3354.

Grell RF (1984). Time of recombination in the Drosophila melanogaster oocyte. III.

Selection and characterization of temperature-sensitive and-insensitive,

recombination-deficient alleles in Drosophila. Genetics 108: 425-443.

Hales KG, Korey CA, Larracuente AM, Roberts DM (2015). Genetics on the Fly: A

Primer on the Drosophila Model System. Genetics 201: 815-842.

Higgins JD, Perry RM, Barakate A, Ramsay L, Waugh R, Halpin C, et al. (2012).

Spatiotemporal Asymmetry of the Meiotic Program Underlies the Predominantly

Distal Distribution of Meiotic Crossovers in Barley. Plant Cell 24: 4096-4109.

Hunter CM (2015). Environmental and Genetic Determinants of Recombination Rate in

Drosophila melanogaster. Ph.D. Thesis, North Carolina State University. 
Kulathinal RJ, Bennett SM, Fitzpatrick CL, Noor MAF (2008). Fine-scale mapping of recombination rate in Drosophila refines its correlation to diversity and divergence. Proc Natl Acad Sci 105: 10051-10056.

Kulathinal RJ, Stevison LS, Noor MAF (2009). The Genomics of Speciation in Drosophila: Diversity, Divergence, and Introgression Estimated Using LowCoverage Genome Sequencing. PLoS Genet 5.

Kuntz SG, Eisen MB (2014). Drosophila Embryogenesis Scales Uniformly across Temperature in Developmentally Diverse Species. PLoS Genet 10.

Mampell K (1943). Genetic Studies in Drosophila pseudoobscura. PhD Thesis Thesis, California Institute of Technology.

Markow TA (2019). Ecological and Evolutionary Genomics: The Cactophilic Drosophila Model System. J Hered 110: 1-3.

McGaugh SE, Heil CSS, Manzano-Winkler B, Loewe L, Goldstein S, Himmel TL, et al. (2012). Recombination Modulates How Selection Affects Linked Sites in Drosophila. PLOS Biol 10: e1001422.

Page SL, Hawley RS (2003). Chromosome Choreography: The Meiotic Ballet. Science 301: 785-789.

Parsons PA (1988). Evolutionary rates: effects of stress upon recombination. Biol J Linn Soc 35: 49-68.

Parsons PA (1991). Biodiversity Conservation Under Global Climatic Change: The Insect Drosophila as a Biological Indicator? Glob Ecol Biogeogr Lett 1: 77-83. Phadnis N (2011). Genetic Architecture of Male Sterility and Segregation Distortion in Drosophila pseudoobscura Bogota-USA Hybrids. Genetics 189: 1001-1009. 
Plough HH (1917). The effect of temperature on crossing over in Drosophila. J Exp Zool

24: 147-209.

Plough HH (1921). Further studies on the effect of temperature on crossing over. $J$ Exp

Zool 32: 187-202.

Pownall K, Taylor H, Altindag UH, Stevison LS (2020). Maternal age alters

recombination rate in Drosophila pseudoobscura. bioRxiv 2020.07.20.212548.

R Core Team (2020). R: A language and environment for statistical computing. $\mathrm{R}$

Foundation for Statistical Computing,: Vienna, Austria.

Ritz KR, Noor MAF, Singh ND (2017). Variation in Recombination Rate: Adaptive or Not? Trends Genet 33: 364-374.

Samuk K, Manzano-Winkler B, Ritz KR, Noor MAF (2020). Natural Selection Shapes

Variation in Genome-wide Recombination Rate in Drosophila pseudoobscura.

Curr Biol 30: 1517-1528.e6.

Scott Chialvo CH, White BE, Reed LK, Dyer KA (2019). A phylogenetic examination of host use evolution in the quinaria and testacea groups of Drosophila. Mol Phylogenet Evol 130: 233-243.

Stevison LS, Noor MAF (2010). Genetic and Evolutionary Correlates of Fine-Scale Recombination Rate Variation in Drosophila persimilis. J Mol Evol 71: 332-345.

Stevison LS, Sefick S, Rushton C, Graze RM (2017). Recombination rate plasticity: revealing mechanisms by design. Philos Trans $R$ Soc B Biol Sci 372: 20160459. 


\section{0 word article summary}

Meiotic recombination contributes to genetic variation by creating different combinations of alleles and is often deemed beneficial for eukaryotic organisms. Different environmental factors such as temperature affect recombination rate. Even though plasticity in Drosophila has been studied extensively, the timing and duration of the events regulating plasticity remain understudied. The sensitive time in peak plasticity was identified as day 6 in $D$. melanogaster. Here, we use D. pseudoobscura, an alpine species, to observe the peak timing in recombination rates in response to developmental temperature stress following 24 hour transfers, we show that that recombination differences peak at day 9 post-mating. 


\section{Figures}

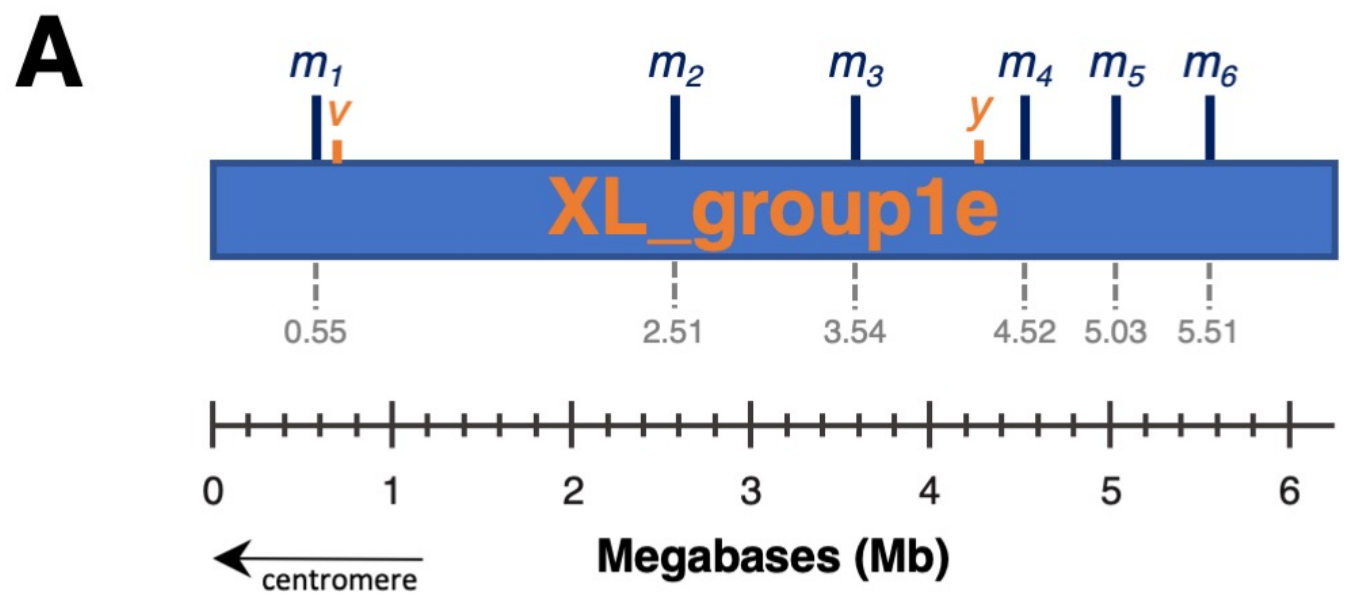

B

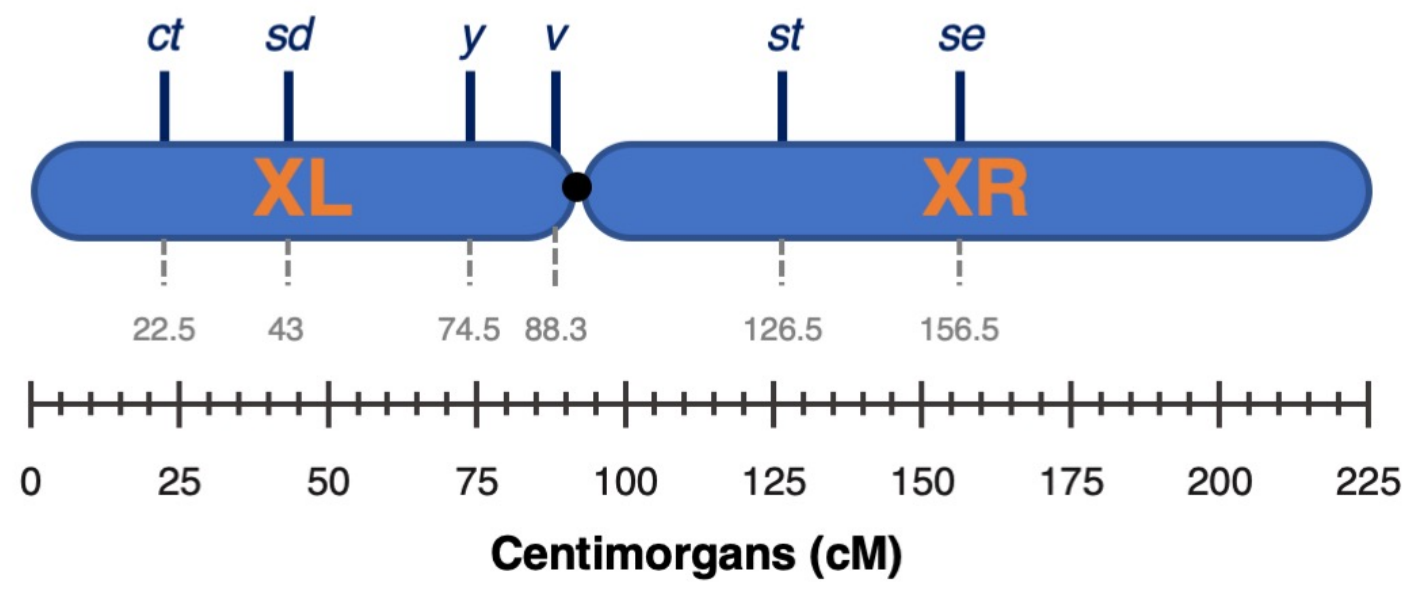

Figure 1. Physical and genetic location of markers used to measure recombination frequency in $\boldsymbol{D}$. pseudoobscura. (A) Physical locations of SNP genotyping markers along $12.5 \mathrm{Mb}$ scaffold "XL_group1e" located on the left arm of the X chromosome $(\mathrm{XL})$. This reverse orientation scaffold covers $62 \%$ of $\mathrm{XL}$, including the mutant markers vermillion and yellow. (B) Genetic map of X chromosome with location of mutant X-linked markers for recombinant screens. The majority of markers were located on the XL. It was later discovered that the presumed vermillion marker in Experiments 1-3 was actually scarlet (also shown). 

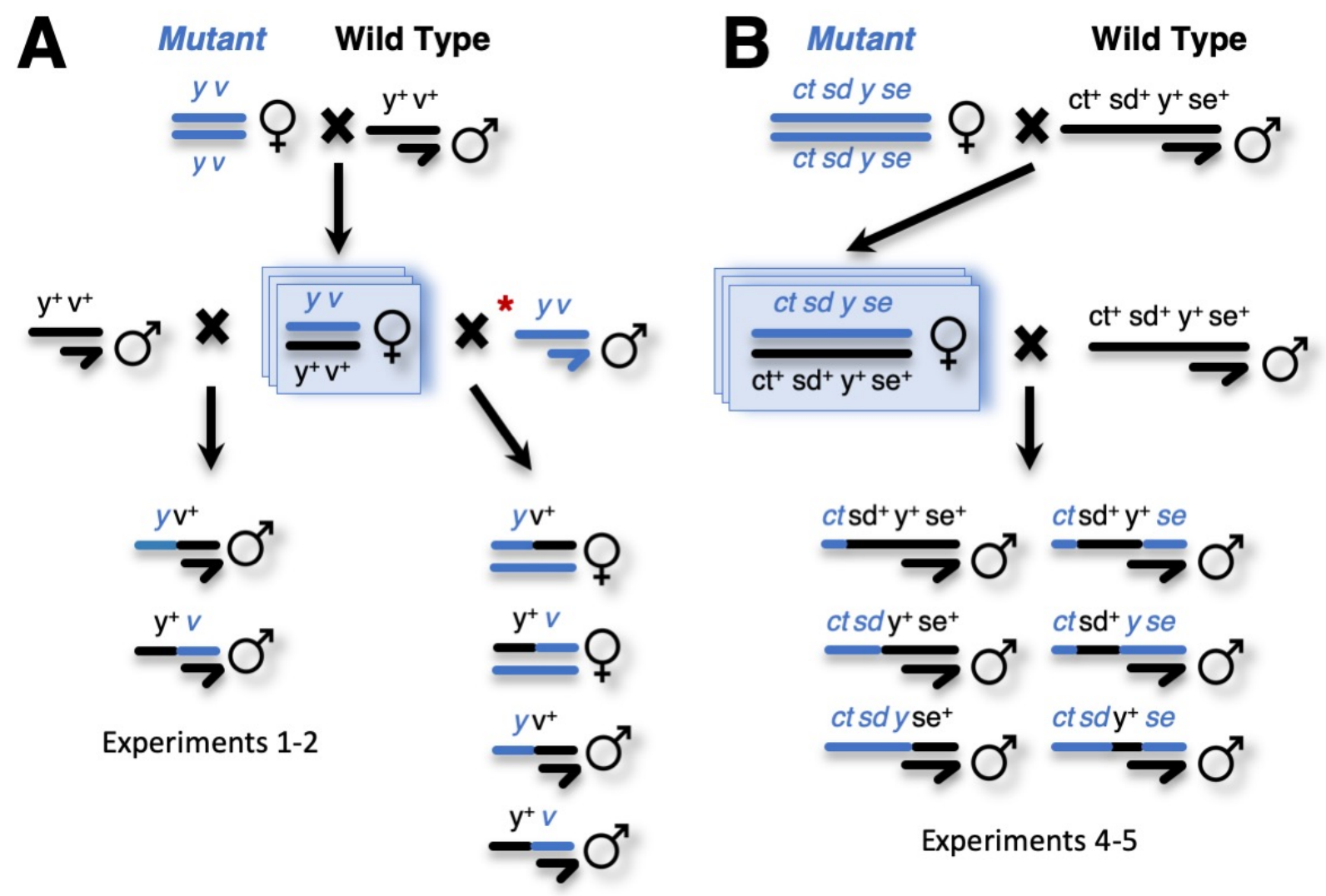

Experiments 4-5

Experiment 3

Figure 2. Crossing scheme for mutant phenotype screens. Females homozygous for mutant markers of two stocks were used to cross to wildtype flies. This $F_{1}$ cross was the unit of replication, as indicated by the stacked boxes, and the resulting female progeny experienced the developmental difference in rearing temperatures as indicated in Table 1. The ID of these crosses were tracked in the resulting backcrosses. In Experiments 13 , the $y-v$ mutant stock and the MV2-25 wildtype stock were used (A). In Experiments 1 and $2, F_{1}$ females were backcrossed to the wildtype stock, whereas in Experiment 3, $F_{1}$ females were backcrossed to the mutant stock (indicated by red asterisk). For Experiments 4-5, the quadruple mutant stock ct-sd-y-se and the SCl_12.2 wildtype stock were used (B). Based on initial screening of male progeny, the marker ct was removed from consideration as it gave unreliable results due to incomplete penetrance. Male progeny were screened for recombination analysis (Eq. 1) and female progeny were included for fecundity analysis (Eq. 2). 


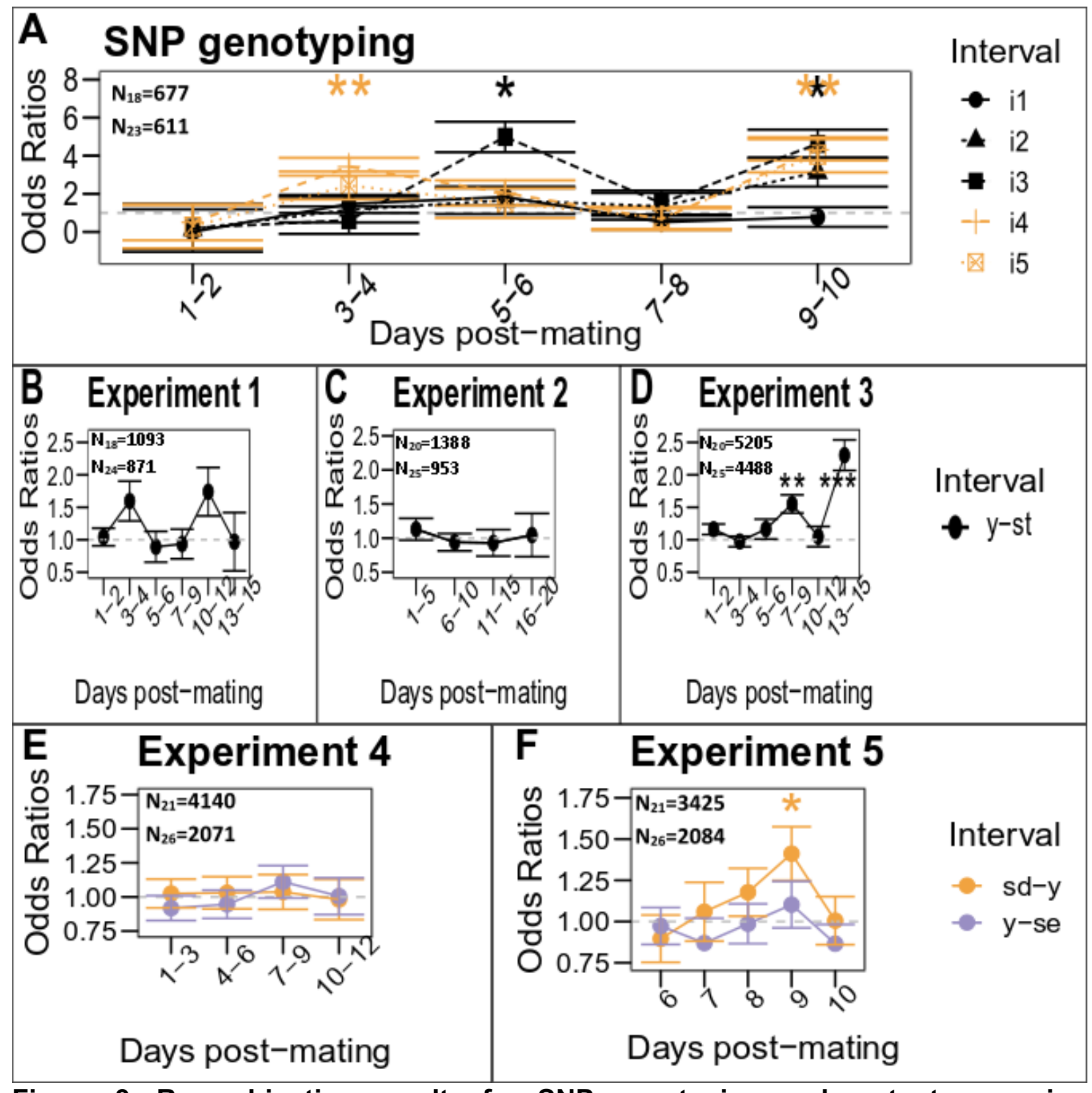

Figure 3. Recombination results for SNP genotyping and mutant screening Experiments 1-5. Recombination frequencies between control and treatment were compared using a fitted generalized linear mixed-effects model (GLMM). Exponentiating the coefficients generated the odds ratio. Odd ratios across experiments were plotted against days post-mating and indicate the odds of having a crossover in high temperature compared to control. (A) Odds ratios for the SNP genotyping markers with the region overlapping the $y$-st interval in black, and the region overlapping $s d-y$ in orange. (B-D) Odds ratios for the double recombination analysis in the $y$-st region, and (E-F) odds ratios for the quadruple recombination analysis in the intervals $s d$-y (orange) and $y$-se (purple). A post hoc test was done to calculate significance for each timepoint between treatment and control with significance indicated via asterisks (see Tables S3 and S4). See Table 1 for additional details regarding differences in experimental design across panels. 


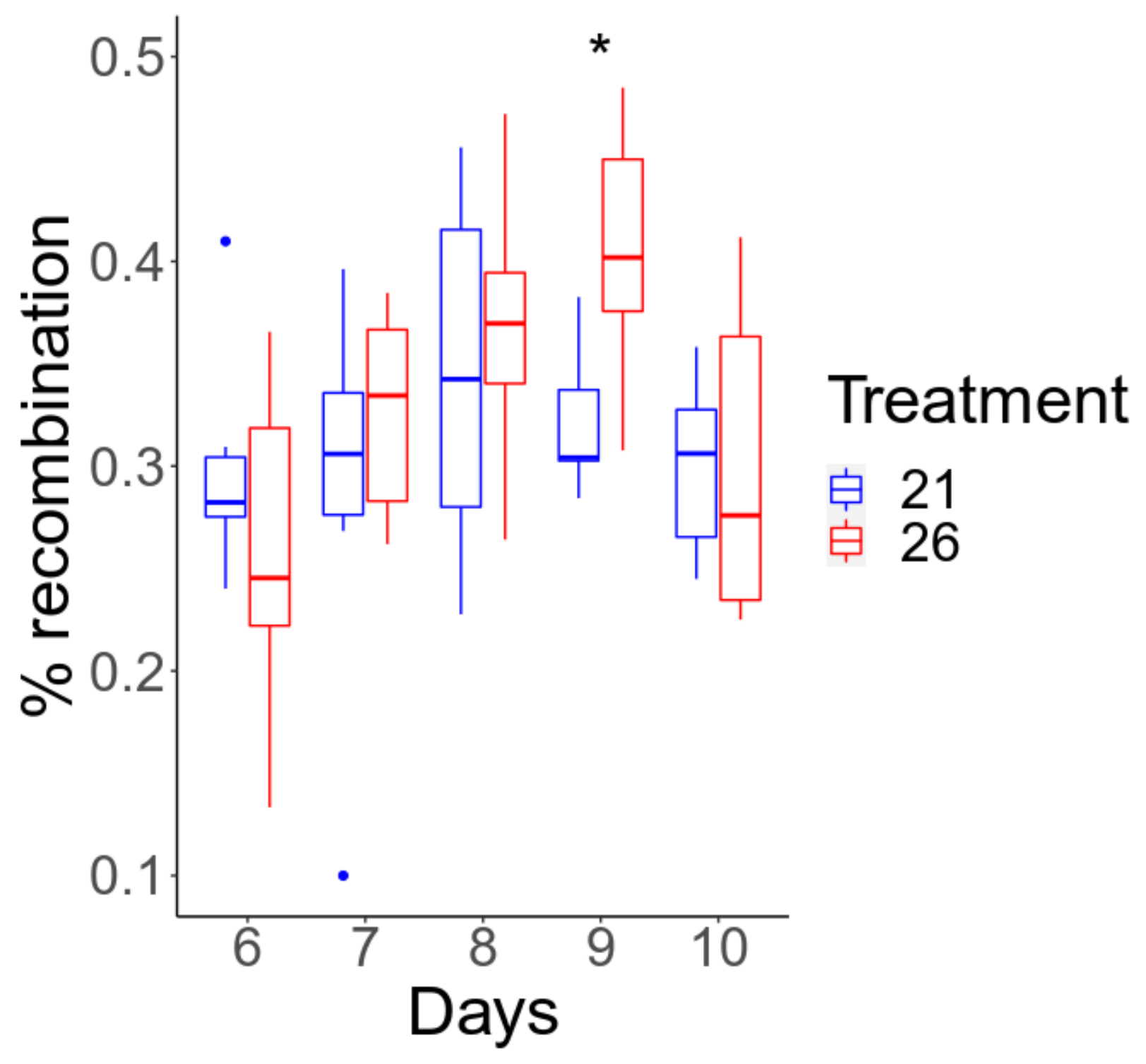

Figure 4. Recombination rates across interval sd-y over $24 \mathrm{~h}$ periods from Experiment 5. As in Figure 3F, day 9 shows a significant difference in recombination rate $\left({ }^{*} \mathrm{p}=0.034\right)$ between the control (blue) and high temperature (red) for the interval between scalloped-yellow. The boxplots show variation in recombination rate among the six replicates per treatment. 


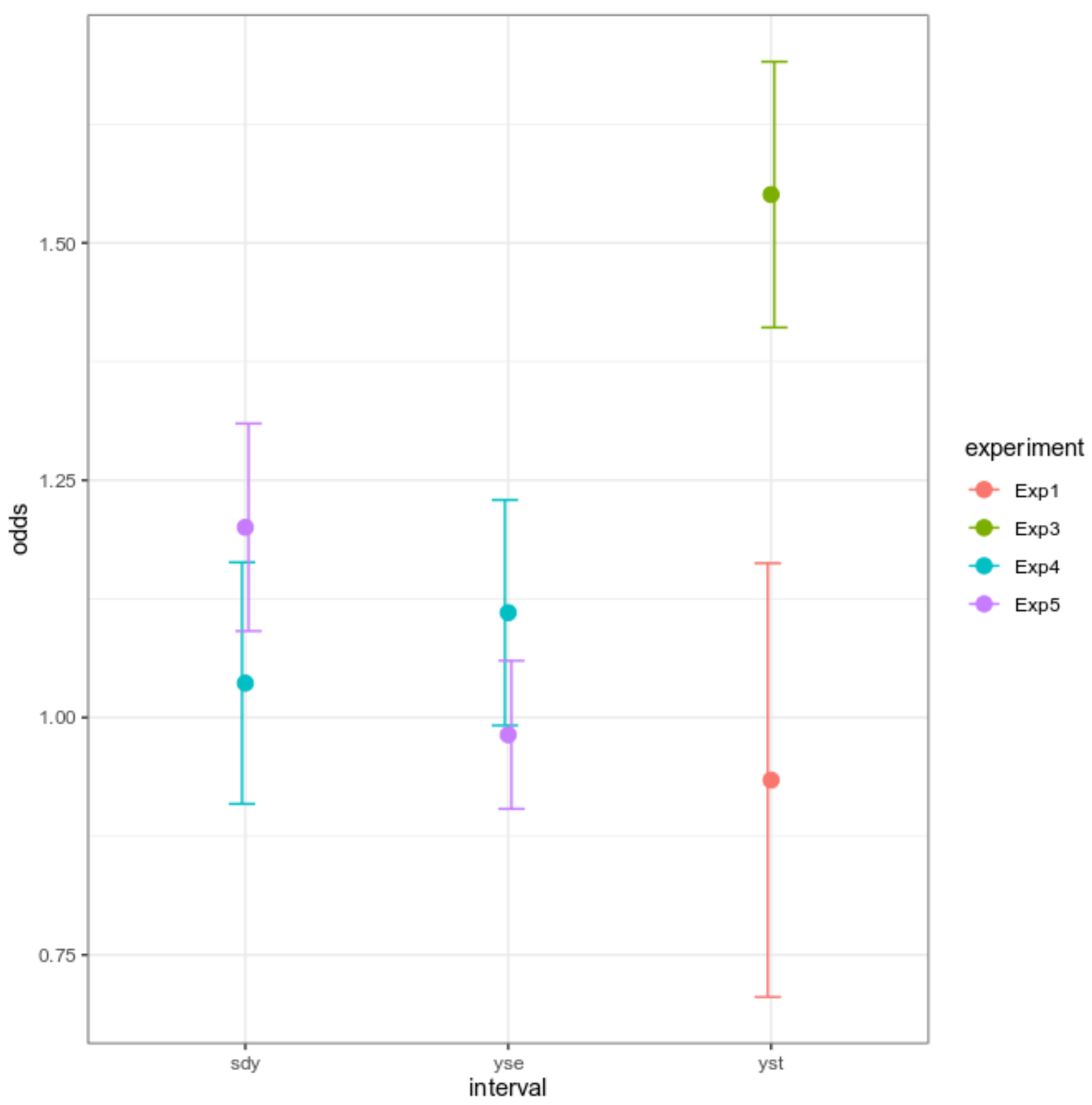

Figure 5. Reproducibility of results between experiments. For days 7-9, odds ratios were compared across experiments for the intervals $y$-sd, $y$-se, and $y$-st. Legend indicates experiments compared by summing across multiple time points for $24 \mathrm{H}$ transfer frequency experiments. 


\section{Tables}

Table 1. Summary of experimental design and results for genotyping experiments to measure the impact of temperature on recombination frequency. For each experiment, a different set of temperatures, transfer frequencies and duration as well as sample sizes were used. *Note: Sample size is based only on the number of individuals targeted for recombination frequency. (e.g. only males were phenotyped for Exp 1, 2, 4, and 5). For fecundity, values are based on all progeny of both sexes for the duration of the experiment but not over the lifetime of each replicate female. For transfer frequency and duration, $\mathrm{H}$ denotes hours and $\mathrm{D}$ denotes days. oP-value from Eq. 2 for Treatment on Fecundity. キP-value from Eq. 1 for Treatment on Recombination Rate. Full Anova tables for both analyses are in Table S1, S2, and S5. ${ }^{\text {aC }}$ rossing scheme matches Figure $2 \mathrm{~A}$. ${ }^{\mathrm{b}}$ Crossing scheme matches Figure 2B.

\begin{tabular}{|c|c|c|c|c|c|c|c|}
\hline & $\begin{array}{c}\text { Transfer } \\
\text { frequency/d } \\
\text { uration }\end{array}$ & $\begin{array}{c}\text { \# rep } \\
\text { cross/tre } \\
\text { atment }\end{array}$ & $\begin{array}{l}\text { Median \# } \\
\text { crosses/ } \\
\text { rep }\end{array}$ & $\begin{array}{c}\text { Temperat } \\
\text { ures }\end{array}$ & $\begin{array}{l}\text { Sample } \\
\text { Size* }\end{array}$ & $\begin{array}{c}\text { Fecun } \\
\text { dity? }\end{array}$ & $\begin{array}{c}\text { Recombinat } \\
\text { on } \neq\end{array}$ \\
\hline $\begin{array}{c}\text { SNP } \\
\text { Genotyping }\end{array}$ & $48 \mathrm{H} / 10 \mathrm{D}$ & $4 / 4$ & $4 / 4$ & $18^{\circ} \mathrm{C} / 23^{\circ} \mathrm{C}$ & $677 / 611$ & $\mathrm{n} / \mathrm{a}$ & 0.29 \\
\hline $\begin{array}{c}\text { Experiment } \\
1 \mathrm{a}\end{array}$ & $24 \mathrm{H} / 15 \mathrm{D}$ & $4 / 4$ & $9 / 8$ & $\begin{array}{c}18^{\circ} \mathrm{C} / 24.5^{\circ} \\
\mathrm{C}\end{array}$ & $1093 / 871$ & 0.40 & 0.47 \\
\hline $\begin{array}{c}\text { Experiment } \\
2 \mathrm{a}\end{array}$ & $120 \mathrm{H} / 20 \mathrm{D}$ & $4 / 5$ & $8 / 6$ & $20^{\circ} \mathrm{C} / 25^{\circ} \mathrm{C}$ & $1388 / 953$ & 0.08 & 0.98 \\
\hline $\begin{array}{c}\text { Experiment } \\
3 \mathrm{a}\end{array}$ & $\begin{array}{c}\text { 48H/6D; } \\
72 \mathrm{H} / 7-15 \mathrm{D}\end{array}$ & $8 / 9$ & $10 / 10$ & $20^{\circ} \mathrm{C} / 25^{\circ} \mathrm{C}$ & $\begin{array}{c}5205 / 448 \\
8\end{array}$ & 0.24 & 0.01 \\
\hline $\begin{array}{c}\text { Experiment } \\
4 b\end{array}$ & $72 \mathrm{H} / 12 \mathrm{D}$ & $5 / 5$ & $5 / 5$ & $21^{\circ} \mathrm{C} / 26^{\circ} \mathrm{C}$ & $\begin{array}{c}4140 / 207 \\
1\end{array}$ & $\begin{array}{c}\text { 5.54E- } \\
11\end{array}$ & $\begin{array}{c}0.76(\mathrm{sd}- \\
\mathrm{y}), 0.91(\mathrm{y}-\mathrm{se})\end{array}$ \\
\hline $\begin{array}{c}\text { Experiment } \\
5 \mathrm{~b}\end{array}$ & $24 \mathrm{H} / 6-10 \mathrm{D}$ & $6 / 6$ & $12 / 12$ & $21^{\circ} \mathrm{C} / 26^{\circ} \mathrm{C}$ & $\begin{array}{c}3425 / 208 \\
4\end{array}$ & $\begin{array}{c}4.49 \mathrm{E}- \\
04\end{array}$ & $\begin{array}{l}0.09(\mathrm{sd}-\mathrm{y}) \\
0.59(\mathrm{y}-\mathrm{se})\end{array}$ \\
\hline
\end{tabular}

